\title{
Ensacamento de cachos na produção, maturação e qualidade dos frutos de bananeiras cultivadas no Estado do Amazonas ${ }^{1}$
}

\author{
Luis Pereira da SILVA FILHO ${ }^{2}$, Adônis MOREIRA ${ }^{3}$
}

\begin{abstract}
RESUMO
O objetivo deste trabalho foi avaliar o efeito do ensacamento de cachos sobre a produção, período de maturação e qualidade dos frutos das cultivares de bananeiras (Thap maeo, FHIA 18, Nanicão 2001 e Prata Zulu). Empregou-se o delineamento experimental inteiramente casualizado em esquema fatorial $4 \mathrm{x} 2$, sendo os fatores: as quatro cultivares conduzidos com cachos ensacados e não ensacados em quatro repetições. O ensacamento foi efetuado com sacos de polietileno impregnados com inseticida organofosforado. As variáveis avaliadas foram: intervalo em dias entre a emergência da inflorescência e a colheita; peso do cacho; peso da penca, diâmetro dos frutos; relação polpa/casca; $\mathrm{pH}$; textura e sólidos solúveis. Nas condições edafoclimáticas locais, na média das cultivares, as variáveis intervalo entre a emergência da inflorescência e a colheita dos cachos, diâmetro dos frutos, relação polpa/casca, peso da penca, $\mathrm{pH}$, textura dos frutos e sólidos solúveis não foram significativamente influenciados pela prática do ensacamento de cachos. O ensacamento diminuiu significativamente a produção da cultivar Prata Zulu.
\end{abstract}

\section{PALAVRAS-CHAVE}

Musa spp., pós-colheita, sacos de polietileno

\section{Bag enclose on the yield, ripening period and fruit quality of banana planted in the Amazonas State, Brazil}

\begin{abstract}
The objective of this work was to evaluated the effect of bag enclose on the yield, ripening period and fruit quality of bananas cultivars (Thap maeo, FHIA 18, Nanicão 2001 and Prata Zulu). A randomized experimental design was employed, in a $4 x 2$ factorial, comprising the four cultivars and two procedures (bagged and not bagged bunches), with four replicates. The bagging was done with bags impregnated with organophosphorated insecticide. The variables analyzed were the time elapsed between the emergence of the inflorescence and the fruit barvest; bunch yield, band yield; pulp diameter; fruit diameter; pulp/rind ratio; $\mathrm{pH}$; texture and soluble solids. In the edaphoclimatic conditions, the results showed, on an average for all cultivars, that the baggin had no significative effect on the emergence of the inflorescence, pulp diameter, fruit diameter, pulp/rind ratio, $p H$, texture and soluble solids. It is noteworthy that even without significant difference, it was verified that, irrespective of cultivar, there was a decrease of the ripening period and a reduction of the bunch weight. The bags enclose reduced by cultivar Prata Zulu yield.
\end{abstract}

KEY-WORDS

Musa spp., post harvest, polyethylene bags

\footnotetext{
${ }^{1}$ Parte da Dissertação de Mestrado submetida, pelo autor, à Universidade Federal do Amazonas - UFAM.

${ }^{2}$ Estudante de pós-graduação da Universidade Federal do Amazonas, Av. Gal. Rodrigo Otávio, 3000, CEP 69077-000, Manaus-AM (irmao@uol.com.br).

${ }_{3}^{3}$ Pesquisador da Embrapa Amazônia Ocidental, Caixa Postal 319, CEP 69011-970, Manaus-AM. Bolsista do CNPq. (adonis@cpaa.embrapa.br).
} 


\section{ACTA \\ AMAZONICA}

ENSACAMENTO DE CACHOS NA PRODUÇÃO, MATURAÇÃO E QUALIDADE DOS FRUTOS DE BANANEIRAS CULTIVADAS NO

ESTADO DO AMAZONAS

\section{INTRODUÇÃO}

Os mercados de exportação de bananas do Brasil são preferencialmente a Argentina e o Uruguai. Porém, essa transação comercial é pouco expressiva, e ainda se verifica a perda na participação do mercado argentino, que tem passado a importar do Equador, devido a melhor qualidade dos frutos (Scarpare Filho, 1996).

As perspectivas de recuperar este mercado e ampliar seus negócios com os países citados, aliadas ao fato de que o Mercosul apresenta-se como uma expectativa do mercado sólido e rentável, é necessário que o Brasil passe a investir em tecnologia de produção, sob o risco de ver declinar a sua modesta cota no mercado sul-americano (Scarpare Filho, 1996).

Gonçalves et al. (1994) salientam sobre a dificuldade de comparar, em nível global, a bananicultura desenvolvida no Brasil com a atividade desenvolvida em bases empresariais, como acontece em outros países, onde há comprometimento com a qualidade do produto.

No Brasil, um dos principais problemas é a qualidade dos frutos produzidos, fato que, certamente, contribui para a baixa participação na exportação do produto. O ensacamento de cachos, no início de seu desenvolvimento na planta, pode ser uma alternativa que venha contribuir para a melhoria da qualidade dos frutos de banana no Brasil. Os benefícios são devidos, às vezes, ao aumento de peso de cacho; à redução de intervalo entre a emergência da inflorescência e à colheita; $\mathrm{e}$, principalmente, a melhoria visual dos frutos tornando-os mais atrativos para o consumidor (Heenan, 1973; Soto Ballestero et al., 1992; Lichtemberg, 1996).

Inúmeros trabalhos mostram que rendimento e qualidade de cachos desenvolvidos no inverno são melhores quando ensacados. As elevações de produtividade são atribuídas ao aumento de peso, à redução entre a emergência da inflorescência e à colheita ou ambos (Robinson, 1995).

Rodrigues et al. (2001), ao estudar a influência do ensacamento do cacho de bananeira Prata-Anã em três ciclos de produção, concluíram que nas características peso do cacho, peso total dos frutos, peso médio dos frutos, peso médio das pencas, comprimento, diâmetro, peso e espessura da casca do fruto central da segunda penca não houve efeito significativo dos tratamentos para os três ciclos avaliados.

Lichtemberg et al. (1998) estudando a cultivar Grande Naine e diferentes tipos de sacos, concluíram que o ensacamento propiciou a produção de cachos mais pesados e de frutos mais longos, com maior diâmetro. O efeito dos diversos tipos de sacos foi variável quanto ao peso das pencas e dos frutos, comprimento dos frutos e danos por frio, tripes da ferrugem $\mathrm{e}$ da erupção (Caliothrips bicinctus, Chaetanaphotrips orchidii eTryphactothrips leneatus) e ponta-do-charuto (Trachysphaera fructigena). Não foi verificado efeito do ensacamento sobre o número de dias do florescimento à colheita.

Costa (1998), trabalhando com Nanicão no município de Tietê, Estado de São Paulo, estudou o efeito do ensacamento no inverno, verão e outono, não encontrando diferenças em relação ao $\mathrm{pH}$ e a relação polpa/casca. No entanto, verificou-se efeito do ensacamento dos cachos no teor de sólidos solúveis e firmeza dos frutos. O ensacamento dos cachos ocasiona a formação de um microclima dentro do saco, com o aumento da temperatura, o que propicia mudanças fisiológicas nos frutos de bananeira (Soto Ballestero, 1992; Lichtemberg, 1996). Além dessas mudanças, existem diferenças de resposta ao ensacamento, de acordo com o clima e entre diferentes cultivares (Soto Ballestero, 1992).

Este trabalho teve como objetivo avaliar o efeito do ensacamento em cachos de banana com sacos de polietileno impregnados com inseticida organofosforado, sobre a produção, período de maturação e qualidade dos frutos das cultivares de bananeiras Prata Zulu, Nanicão 2001, Thap maeo e FHIA 18 cultivadas em Latossolo Amarelo distrófico de terra firme no Estado do Amazonas.

\section{MATERIAL E MÉTODOS}

O experimento foi conduzido em Latossolo Amarelo distrófico de textura argilosa com as seguintes características químicas: $\mathrm{pH}$ em $\mathrm{H}_{2} \mathrm{O}=4,3 ; \mathrm{P}$ - Mehlich $1=1,8 \mathrm{mg} \mathrm{dm}^{-3} ; \mathrm{K}$ Mehlich $1=47,7 \mathrm{mg} \mathrm{dm}^{-3} ; \mathrm{Ca}-\mathrm{KCl} 1,0 \mathrm{~mol} \mathrm{~L}^{-1}=0,24 \mathrm{cmol}^{-}$ $\mathrm{dm}^{-3} ; \mathrm{Mg}-\mathrm{KCl} 1,0 \mathrm{~mol} \mathrm{~L}^{-1}=0,92 \mathrm{cmol}_{\mathrm{c}} \mathrm{dm}^{-3} ; \mathrm{Al}-\mathrm{KCl} 1,0 \mathrm{~mol}$ $\mathrm{L}^{-1}=1,45 \mathrm{cmol}_{c} \mathrm{dm}^{-3} ; \mathrm{H}+\mathrm{Al}=8,04 \mathrm{cmol}_{\mathrm{c}} \mathrm{dm}^{-3} ; \mathrm{CTC}=9,32$ $\mathrm{cmol}_{\mathrm{c}} \mathrm{dm}^{-3}$; Boro - Mehlich $1=0,41 \mathrm{mg} \mathrm{dm}^{-3} ; \mathrm{Cu}=0,29 \mathrm{mg}$ $\mathrm{dm}^{-3} ;$ Fe - Mehlich $1=333 \mathrm{mg} \mathrm{dm}^{-3} ;$ Mn - Mehlich $1=5,15 \mathrm{mg}$ $\mathrm{dm}^{-3} ; \mathrm{Zn}$ - Mehlich $1=0,68 \mathrm{mg} \mathrm{dm}^{-3}$, localizado no campo Experimental da Embrapa Amazônia Ocidental, nas coordenadas geográficas 3 ${ }^{\circ} 8^{\prime} 5^{\prime \prime} \mathrm{LS}$ e $59^{\circ} 52^{\prime}$ ' LW, no município de Manaus, Estado do Amazonas.

O delineamento experimental utilizado foi o inteiramente casualizado em fatorial (4x2), sendo quatro cultivares [Prata Zulu (AAB), FHIA 18 (AAAB), Nanicão 2001 (AAA) e Thap Maeo $(\mathrm{AAB})]$ e dois tipos de manejo (cachos ensacados e não ensacados), com quatro repetições. As bananeiras avaliadas estavam no segundo (Nanicão 2001 e Thap maeo) e terceiro ciclos de produção (Prata Zulu e FHIA 18). As touceiras foram manejadas por desbastes deixando a mãe, filha e neta, sendo os outros brotos retirados.

A adubação consistiu na aplicação de 70 gramas de uréia e 270 gramas de cloreto de potássio a cada dois meses. No mesmo período foram aplicados de uma só vez 150 gramas de superfosfato simples, 50 gramas de FTE BR 12 - fritas $(1,8 \%$ de B, $0,8 \%$ de $\mathrm{Cu}, 3,0 \%$ de Fe, $2,0 \%$ de $\mathrm{Mn}, 0,1 \%$ de Mo e $9,0 \%$ de Zn) e 100 gramas de sulfato de magnésio (Pereira et al., 2002).

Foram utilizados no ensacamento sacos de plástico perfurados de cor azul transparente com densidade de $1,0 \mathrm{~g}$ $\mathrm{mL}^{-1}$, impregnados com inseticida organofosforado, com as seguintes características químicas: $10 \mathrm{~g} \mathrm{~kg}^{-1}$ deCloripirofós e $190 \mathrm{~g} \mathrm{~kg}^{-1}$ de inertes, com meia vida em solução tampão de fosfato a $25^{\circ}$ e pH 5,0 de 63 dias. Optou-se por esse tipo de saco, que segundo Lima (2002), é o mais recomendado para regiões com alta incidência de pragas. 


\section{ACTA \\ AMAZONICA}

ENSACAMENTO DE CACHOS NA PRODUÇÃO,

MATURAÇÃO E QUALIDADE DOS FRUTOS DE BANANEIRAS

CULTIVADAS NO ESTADO DO AMAZONAS
No ensacamento foi utilizado um implemento que fixa o saco no engaço do saco através de um anel de borracha, permanecendo até o final do desenvolvimento. Os cachos foram ensacados no início do florescimento. Na colheita foram analisadas as seguintes variáveis: peso do cacho, intervalo em dias entre a emergência da inflorescência e a colheita (E-C), diâmetro da polpa, relação polpa/casca, pH - acidez, textura e sólidos solúveis totais (Santos \& Chitarra, 1998).

Os dados foram submetidos à análise de variância (teste F), teste de comparação de contraste de médias (Tukey a 5\% de probabilidade), conforme metodologia descrita por Pimentel Gomes (1990).

\section{RESULTADOS E DISCUSSÃO}

O intervalo de emergência da inflorescência e a colheita (EC) não apresentou interação em relação ao ensacamento $x$ cultivar, havendo diferença somente entre as cultivares (Tabela 1). Tal inferência não corrobora os resultados obtidos por Salomão (1995) e Costa et al. (2002), que nas condições edafoclimáticas do Estado de São Paulo obtiveram redução significativa entre o período de emergência da inflorescência até a colheita no período de verão.

A diferença entre os resultados obtidos e os encontrados na literatura ocorre, possivelmente, devido à amplitude térmica existente entre o dia e a noite, que nas condições do presente trabalho foi de aproximadamente $3^{\circ} \mathrm{C}$, enquanto que em São Paulo essa diferença, no verão, é de $8^{\circ} \mathrm{C}$ (Costa et al., 2002). Nessas condições, o saco de polietileno atua como condicionador térmico, o mesmo não ocorre nos cachos das bananeiras do presente estudo, em que a temperatura permaneceu constante. Outro fator que deve ter interferido nos resultados são os critérios utilizados na definição do intervalo entre a emergência da inflorescência e a colheita, cuja determinação pode ser subjetiva (Galán Saúco et al., 1996).

O ciclo de desenvolvimento dos cachos ocorreu nos meses mais quentes do ano (Figura 1), mesmo não apresentando diferenças estatísticas ( $\left.\mathrm{p}^{3} 0,05\right)$, o E-C foi menor nos cachos ensacados. Johns \& Scott (1989) comprovaram, mediante comparação de vários tipos de sacos, a ocorrência de aumento de temperatura dentro desses sacos, sendo mais elevadas no verão.

É interessante ressaltar que o efeito do ensacamento depende tanto da época de realização, quanto das condições climáticas do período. De acordo com Costa (1998), a melhor época para a redução no intervalo entre cachos ensacados nas condições climáticas do Estado de São Paulo é a de maior intensidade de chuvas e maiores temperaturas. A temperatura média entre os meses da condução do experimento foi de $28,3^{\circ} \mathrm{C}$ (Figura 1), estando este valor próximo dos indicados por Stover \& Simmonds (1987) que é entre $29^{\circ} \mathrm{C} \mathrm{e} 30^{\circ} \mathrm{C}$.

Na comparação entre as cultivares, observa-se que a cultivar Nanicão 2001 foi a que apresentou menor intervalo entre a inflorescência e a colheita (Tabela 1). Além do aspecto genético, a temperatura pode ter sido um dos fatores que influenciaram
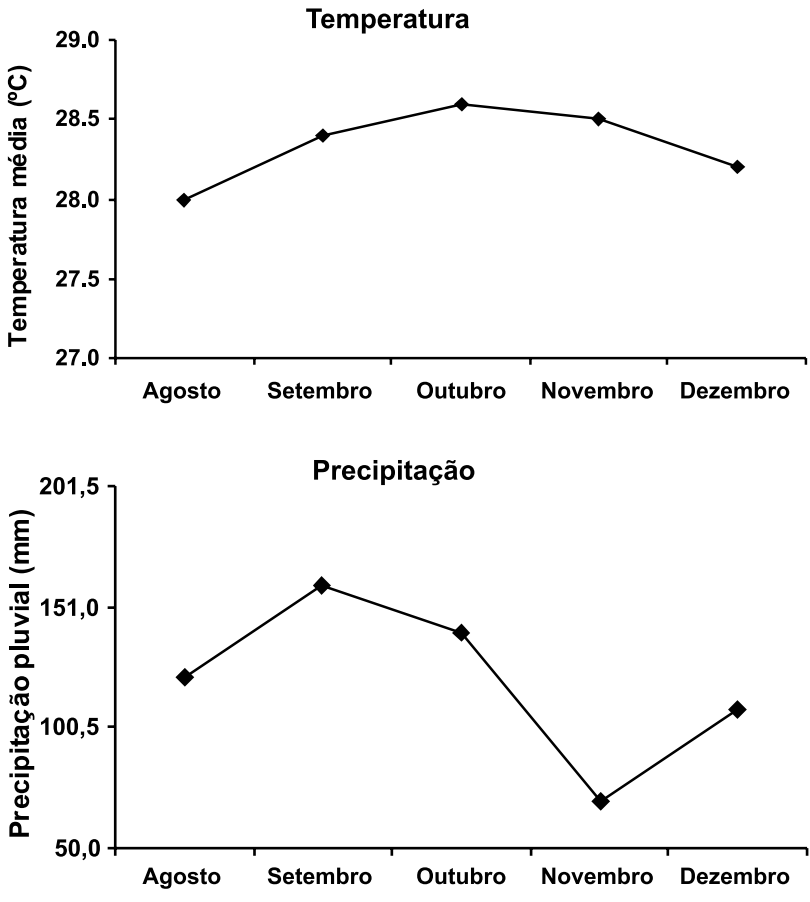

Figura 1 - a) Temperatura média mensal $\left({ }^{\circ} \mathrm{C}\right)$ e b) precipitação pluvial $(\mathrm{mm})$ nos meses de agosto, setembro, outubro, novembro e dezembro de 2003, na estação experimental da Embrapa Amazônia Ocidental, localizada na rodovia AM 010, Km 29, Manaus - AM.

o crescimento e desenvolvimento dos frutos dessa cultivar.

Exceto o mês de novembro, que teve precipitação pluvial média de 69,5 mm, os demais meses (agosto, setembro, outubro e dezembro) tiveram precipitação média mensal acima de $100 \mathrm{~mm}$ (Figura 1). Segundo Soto Ballestero et al. (1992), uma precipitação mínima mensal de $100 \mathrm{~mm}$ a $180 \mathrm{~mm}$ e bem distribuída tem sido aceita como adequada para o bom desempenho da cultura.

Verifica-se na Tabela 1 que o ensacamento de cacho afetou o peso do cacho na cultivar Prata Zulu, havendo diferenças somente entre as cultivares. Quando se observa em termos de produção por hectare (espaçamento $3 \times 2$, correspondendo a 1667 plantas), houve um incremento médio de 10,0 toneladas na cultivar Prata Zulu no tratamento não ensacado.

O efeito do ensacamento sobre a variável peso dos cachos, difere dos encontrados por Cann (1965) e Robinson \& Nel (1984), que detectaram uma elevação na produtividade de $25 \%$ e 16\%, respectivamente com a prática do ensacamento.

Sampaio \& Simão (1970), Daniels et al. (1992), Salomão (1995) e Oliveira et al. (1998) não encontraram diferenças em relação a massa de cachos ensacados e não ensacados. Nota-se nesses trabalhos que, dependendo do local, o ensacamento pode ou não incrementar a produção; o único consenso é que esse procedimento melhora consideravelmente $\mathrm{o}$ aspecto externo do fruto. 


\section{ACTA AMAZONICA}

ENSACAMENTO DE CACHOS NA PRODUÇÃO, MATURAÇÃO E QUALIDADE DOS FRUTOS DE BANANEIRAS CULTIVADAS NO ESTADO DO AMAZONAS
À semelhança do que ocorreu com o intervalo entre a emergência da inflorescência e a colheita, não houve diferenças significativas no diâmetro da segunda penca na interação manejo x cultivar (Tabela 1). Observou-se que, exceto a cultivar Nanicão 2001, houve redução, porém não significativa, com o ensacamento, chegando esse percentual a $11,4 \%$ na cultivar Prata Zulu. Em experimentos conduzidos por Costa (1998), ocorreu redução média, também não significativa, de 5\% no fruto da segunda penca inferior.
Observa-se que o pequeno aumento, mesmo não significativo, do diâmetro dos frutos ensacados da cultivar Nanicão 2001 concorda com os obtidos por Lichtemberg et al. (1998), que, trabalhando com Grande Naine (mesmo grupo genômico) nas condições edafoclimáticas de Itajaí, Santa Catarina, verificaram que o ensacamento propiciou a produção de frutos com maior diâmetro.

A relação polpa/casca diferiu estatisticamente entre as cultivares Thap maeo, Prata Zulu e Nanicão 2001 não ensacadas,

Tabela 1 - Intervalo médio entre a emergência da inflorescência e a colheita, peso do cacho, diâmetro dos frutos, relação polpa/ casca, pH da polpa (acidez), textura da polpa e sólidos solúveis (grau brix) em bananas cultivadas em Manaus, AM. 2003.

\begin{tabular}{|c|c|c|c|c|c|}
\hline \multirow{2}{*}{ Tratamentos } & \multicolumn{5}{|c|}{ Cultivares } \\
\hline & Prata Zulu & FHIA 18 & Nanicão 2001 & Thap maeo & Média \\
\hline & \multicolumn{5}{|c|}{ Intervalo médio entre a emergência da inflorescência e a colheita, dias $(C V \%=6,36)$} \\
\hline Ensacado & 107aA1 & $109 a A$ & $92 \mathrm{aB}$ & $101 \mathrm{aAB}$ & $102 a$ \\
\hline Não ensacado & $117 \mathrm{aA}$ & $109 a A B$ & $99 \mathrm{aB}$ & $103 \mathrm{aB}$ & $107 a$ \\
\hline \multirow[t]{2}{*}{ Média } & $112 \mathrm{~A}$ & $109 A B$ & $95 \mathrm{C}$ & $102 B C$ & \\
\hline & \multicolumn{5}{|c|}{ Peso do cacho, quilogramas $(\mathrm{CV} \%=14,75)$} \\
\hline Ensacado & 30bA & $35 a A$ & $19 \mathrm{aB}$ & $30 \mathrm{aA}$ & $28 a$ \\
\hline Não ensacado & $36 a A$ & $37 a A$ & $17 a B$ & $31 \mathrm{aA}$ & $30 a$ \\
\hline \multirow[t]{2}{*}{ Média } & $33 \mathrm{~A}$ & $36 \mathrm{~A}$ & $18 \mathrm{~B}$ & $30 \mathrm{~A}$ & \\
\hline & \multicolumn{5}{|c|}{ Peso da penca, quilogramas $(\mathrm{CV} \%=17,74)$} \\
\hline Ensacado & $2,41 \mathrm{aB}$ & $3,59 \mathrm{aA}$ & $2,69 \mathrm{aB}$ & $2,50 \mathrm{aB}$ & $2,80 a$ \\
\hline Não ensacado & $2,62 \mathrm{aB}$ & $3,65 a A$ & $2,62 \mathrm{aB}$ & $2,08 \mathrm{aB}$ & $2,74 a$ \\
\hline \multirow[t]{2}{*}{ Média } & $2,52 B$ & $3,62 \mathrm{~A}$ & $2,66 \mathrm{~B}$ & $2,29 B$ & \\
\hline & \multicolumn{5}{|c|}{ Diâmetro dos frutos, mm $(\mathrm{CV} \%=10,29)$} \\
\hline Ensacado & $35,35 a A$ & $40,42 \mathrm{aA}$ & $36,45 a \mathrm{~A}$ & $38,16 a A$ & $37,60 a$ \\
\hline Não ensacado & $39,91 \mathrm{aA}$ & $41,58 \mathrm{aA}$ & $35,39 a A$ & $39,83 a A$ & $39,18 a$ \\
\hline \multirow[t]{2}{*}{ Média } & $37,63 \mathrm{~A}$ & $40,00 \mathrm{~A}$ & $35,92 \mathrm{~A}$ & $39,00 \mathrm{~A}$ & \\
\hline & \multicolumn{5}{|c|}{ Relação polpa/casca $(C V \%=18,05)$} \\
\hline Ensacado & $6,65 a A$ & $2,62 \mathrm{aB}$ & $3,84 \mathrm{bB}$ & $5,81 \mathrm{aAB}$ & $4,73 a$ \\
\hline Não ensacado & $7,17 a A$ & $2,14 \mathrm{aB}$ & $6,83 a \mathrm{~A}$ & $5,77 a A$ & $5,48 a$ \\
\hline \multirow[t]{2}{*}{ Média } & $6,91 \mathrm{~A}$ & $2,38 \mathrm{C}$ & $5,33 \mathrm{~A}$ & $5,79 \mathrm{~A}$ & \\
\hline & \multicolumn{5}{|c|}{$\mathrm{pH}$ da polpa -acidez $(\mathrm{CV} \%=2,86)$} \\
\hline Ensacado & $4,52 \mathrm{aB}$ & $4,47 a B$ & $4,78 \mathrm{aA}$ & $4,30 \mathrm{aC}$ & $4,52 a$ \\
\hline Não ensacado & $4,65 \mathrm{aB}$ & $4,46 \mathrm{aC}$ & $4,82 \mathrm{aA}$ & 4,36aD & $4,57 a$ \\
\hline \multirow[t]{2}{*}{ Média } & $4,58 \mathrm{~B}$ & $4,47 B C$ & $4,80 \mathrm{~A}$ & $4,33 C$ & \\
\hline & \multicolumn{5}{|c|}{ Textura, Newton $(C V \%=21,34)$} \\
\hline Ensacado & $22,08 \mathrm{aA}$ & $16,08 \mathrm{aA}$ & $18,69 \mathrm{aA}$ & $21,52 \mathrm{aA}$ & $19,59 a$ \\
\hline Não ensacado & $20,95 \mathrm{aA}$ & $14,16 \mathrm{aA}$ & $16,08 \mathrm{aA}$ & $16,68 \mathrm{aA}$ & $17,47 a$ \\
\hline \multirow[t]{2}{*}{ Média } & $21,52 \mathrm{~A}$ & $15,12 B$ & $17,38 \mathrm{AB}$ & $20,10 A B$ & \\
\hline & \multicolumn{5}{|c|}{ Sólidos solúveis, grau brix (CV \% = 20,17) } \\
\hline Ensacado & $16,05 \mathrm{aA}$ & $12,22 \mathrm{aAB}$ & $12,90 \mathrm{aAB}$ & $10,65 \mathrm{aB}$ & $12,95 a$ \\
\hline Não ensacado & $12,22 \mathrm{aA}$ & $14,25 \mathrm{aA}$ & $13,27 a A$ & $13,42 \mathrm{aA}$ & $13,29 a$ \\
\hline Média & $14,13 \mathrm{~A}$ & $13,23 \mathrm{~A}$ & $13,08 \mathrm{~A}$ & $12,03 \mathrm{~A}$ & \\
\hline
\end{tabular}

'Médias seguidas por letras distintas minúsculas na mesma coluna e maiúscula na mesma linha dentro de cada cultivar e de cada variável diferem entre si ao nível de $5 \%$ significância pelo teste de Tukey. 


\section{ACTA \\ AMAZONICA}

ENSACAMENTO DE CACHOS NA PRODUÇÃO,

MATURAÇÃO E QUALIDADE DOS FRUTOS DE BANANEIRAS

CULTIVADAS NO ESTADO DO AMAZONAS sendo menor na FHIA 18. No tratamento ensacado as cultivares Thap maeo e Prata Zulu apresentaram as maiores relações (Tabela 1). Com relação ao ensacamento ou não dos cachos, observa-se diferença significativa somente na cultivar 'Nanicão 2001' (Tabela 1). Os resultados com a Thap maeo, FHIA $18 \mathrm{e}$ Prata Zulu corroboram os de Costa (1998), que também não encontrou diferenças significativas entre cachos ensacados e não ensacados. Independentemente da cultivar, as relações ficaram acima das relatadas por Barnell (1941) e Costa (1998), cujas a variações ficaram entre 1,2-1,6 e 1,5-1,6, respectivamente.

Para a variável peso da penca, também não foi encontrada diferença estatística $\left(\mathrm{p}^{3} 0,05\right)$ entre os tratamentos (Tabela 1). Tal resultado corrobora os de Rodrigues et al. (2001) e Costa et al. (2002) que, medindo a influência do ensacamento do cacho na produção de frutos de bananeira da cultivar Prata-anã irrigada e Grande Naine, também não encontraram diferenças significativas. Os resultados encontrados no presente trabalho estão acima ou próximos dos valores obtidos por Gasparotto et al. (2002) trabalhando com as mesmas cultivares, que são: 1,5 quilogramas (Thap maeo), 2,1 quilogramas (Prata Zulu), 2,8 quilogramas (FHIA 18) e 3,0 quilogramas (Nanicão 2001).

$\mathrm{O} \mathrm{pH}$ da polpa não foi afetado pelos tratamentos, não havendo interação ensacamento x cultivar (Tabela 1). As médias encontradas estão abaixo da faixa indicada por Bleinroth (1985), que considera aceitável a variação de 5,0 a 5,6 para frutos verdes. A acidez nos frutos tende a ser mais elevada à medida que diminui a temperatura. Esse comportamento também tem relação com as características varietais dos frutos (Claypool \& Davis, 1959).

Em pesquisa com pêssego, os mesmos autores verificaram uma tendência de diminuição na acidez à medida que a temperatura de armazenamento aumentava de $0^{\circ}$ a 5,6 $6^{\circ}$. Estes estudos inferem que as perdas de íons hidrogênio ocorrem em decorrência da temperatura antes do processo de amadurecimento e que as variações nas concentrações de $\mathrm{H}^{+}$ podem ocorrer devido a eventuais condições climáticas na época de colheita.

Como ocorreu pequena variação na temperatura fora e dentro do saco, esta não afetou o pH da polpa. Na média dos tratamentos ensacados e não ensacados (Tabela 1), observouse que a cultivar Nanicão 2001 foi a que apresentou menor acidez $(4,80)$, enquanto que a Thap maeo foi a que apresentou a maior (4,33). Verifica-se que essas cultivares são grupos genômicos diferentes, ou seja, a cultivar Nanicão 2001 é triplóide AAA, enquanto que a Thap maeo é uma triplóide AAB.

A textura da polpa não foi afetada significativamente pelo ensacamento (Tabela 1). Apesar disso, a cultivar Prata Zulu foi a que apresentou maior textura de polpa (22,1 Newton). Segundo Ryugo (1988), as variações na textura ocorrem somente entre cultivares distintas. Frutos com maior tamanho, com células alongadas, as quais apresentam paredes celulares finas e com maiores espaços entre elas, são propensos a serem mais macios. Isso se justifica quando se observa que as cultivares FHIA 18 e a Nanicão 2001 apresentam as menores texturas da polpa.
Com relação aos sólidos solúveis, pode se observar que não ocorreram diferenças significativas entre os tratamentos ensacados e não ensacados (Tabela 1). Foi observado que o tratamento ensacado a Thap maeo apresentou a menor quantidade de sólidos solúveis, enquanto que a cultivar Prata Zulu apresentou o maior teor. Os sólidos solúveis encontramse dissolvidos na polpa e apresentam a tendência de aumentar a maturação (Chitarra \& Chitarra, 1990). De acordo com Bleinroth (1985), os sólidos solúveis aumentam com a maturação da fruta, por causa da degradação do amido. As diferenças observadas, provavelmente, são devidas ao estado de maturação das próprias frutas no momento da análise.

\section{CONCLUSÕES}

1) O ensacamento de cacho não interferiu no intervalo entre a emergência da inflorescência e colheita do cacho, diâmetro dos frutos, relação polpa casca, peso da penca, $\mathrm{pH}$, textura dos frutos e sólidos solúveis totais.

2) O ensacamento de cachos propiciou redução significativamente na produção da cultivar Prata Zulu.

\section{AGRADECIMENTOS}

Às empresas Teczet Indústria e Comércio Ltda e Agricur defensivos agrícolas, pelas doações dos equipamentos de fixação de bolsas plásticas e sacos de polietileno, respectivamente e aos funcionários da Embrapa Amazônia Ocidental Ednilson e Raimundo César pelo auxílio na condução do experimento.

\section{BIBLIOGRAFIA CITADA}

Barnell, H.R. 1941. Studies on tropical fruits; carbohydrate metabolism of the banana fruit during storage at $53^{\circ} \mathrm{F}$ and ripening at $68^{\circ} \mathrm{F}$. Annals of Botany, 5(20): 608-645, 1941.

Bleinroth, E.W. 1985. Matéria-prima. In: Instituto de Tecnologia de Alimentos. Banana - Cultura, matéria-prima, processamento e aspectos econômicos. Campinas: ITAL, p. 133-196.

Cann, H.J. 1965. Banana growing: plantation practices. Agricultural Gazette on New South Wales, 76(11): 672-678.

Chitarra, M.I.F.; Chitarra, A.B. 1990. Pós-colbeita de frutos e bortaliças: fisiologia e manuseio. ESAL/FAEPE. Lavras. 320p.

Claypool, L.L.; Davis, L.D. 1959. The effect of could and modified atmosphere storage on the canning quality of cling peaches. Food Technology, 13(3): 208-311.

Costa, J.N.M. 1998. Proteção de cachos de bananeira (Musa $\mathrm{sp}$. AAA) em diferentes épocas e periodos, após a emergência da inflorescência. 72f. Dissertação (Mestrado em Agronomia) - Escola Superior de Agricultura "Luiz de Queiroz", Universidade de São Paulo, Piracicaba.

Costa, J.N.M.; Scarpare Filho, J.A.; Kluge, R.A. 2002. Efeito do ensacamento de cachos de banana 'Nanicão' na produção 


\section{ACTA AMAZONICA}

ENSACAMENTO DE CACHOS NA PRODUÇÃO, MATURAÇÃO E QUALIDADE DOS FRUTOS DE BANANEIRAS CULTIVADAS NO ESTADO DO AMAZONAS e no intervalo entre inflorescência e colheita. Pesquisa Agropecuária Brasileira, 37(11): 1575-1580.

Daniells, J.W.; Lisle, A.T.; O'Farrel, P.J. 1992. Effect of bunchcovering methods on maturity bronzing, yield, and fruit quality of bananas in North Queensland. Australian Journal Experimental Agriculture, 32(1): 122 -125.

Galán Saúco, V.G.; Cabrera, J.C.; Gomes Leal, P.M. 1996. The evolution of different bunch covers for banana (Musa acuminata) in the Canary Islands. Fruits, 51(1): 13-24.

Gasparotto, L.; Pereira, J.C.R.; Pereira, M.C.N. 2002. Cultivares de bananeiras resistentes à sigatoka negra. Fitopatologia Brasileira, 27(Suplemento): 220.

Gonçalves, J.S.; Perez, L.H.; Souza, S.A.M. 1994. Mercado internacional e produção de banana: a estrutura produtiva e comercial do complexo bananeiro mundial. Agricultura em São Paulo, 41(1): 161-188.

Heenan, D.P. 1973. Bunch covers for bananas in the Northern District. Papua New Guinea Agricultural Journal, 24(1): 156-161.

Johns, G.G.; Scott, K.J. 1989. Delayed harvesting of banana of bananas with 'sealed' covers on bunches 2 : effect on fruit yield and quality. Australian Journal of Experimental Agriculture, 29(2): 727-733.

Lichtemberg, L.A. 1996. Ensacamento do cacho de bananas no campo. Informativo da Sociedade Brasileira de Fruticultura, 1(3): 8-11.

Lichtemberg, L.A.; Hinz, R.H. Malburg, J.L.; Schitt, A.T.; Lichtemberg, S.H.; Stucker, H. 1998. Efeito do ensacamento do cacho sobre componentes da produção e da qualidade de banana: in: Congresso Brasileiro de Fruticultura, 15. Poços de Caldas, 1998. Resumos... SBF, Poços de Caldas, p. 136.

Lima, M.B. 2002. O ensacamento do cacho da bananeira. Cruz das Almas: Embrapa Mandioca e Fruticultura. 2p

Oliveira, L.A.; Souto, R.F.; Rodrigues, M.G.V.; Menegucci, J.L.P. 1998. Efeito poda da última penca do cacho da prata anã, na produtividade e qualidade dos frutos. In: Congresso Brasileiro de Fruticultura, 15., Poços de Caldas, Anais... Lavras, Sociedade Brasileira de Fruticultura, p.134.

Pereira, M.C.N.; Gasparotto, L.; Pereira, J.C.R.; Lopes, C.M.D. 2002. Manejo da cultura da bananeira no Estado do Amazonas. Manaus: Embrapa Amazônia Ocidental, p14. (Circular Técnica, 10).

Pimentel Gomes, F. 1990. Curso de estatística experimental. Piracicaba: Editora Nobel, 468p.
Robinson, J.C. 1995. Systems of cultivation and management. In: Gowen, S. (Ed.). Bananas and Plantains. Chapman \& Hall. London. p.15-65.

Robinson, J.C.; Nel, D.J. 1984. Banana bunchs covers in winter. Citrus and Subtropical Fruit Research, v.138, p.5-6.

Rodrihues, M.G.V.; Souto, R.F.; Menegucci, J.L.P. 2001. Influência do ensacamento do cacho na produção de frutos da bananeira - 'Prata-Anã' irrigada na Região Norte de Minas Gerais. Revista Brasileira de Fruticultura, 23(3): 559-562.

Ryugo, K. 1988. Fruit culture: its science and art. John Willey. New York. p.107-116.

Salomão, L.C.C 1995. Efeitos do envoltório plástico no desenvolvimento e na maturação pós-colheita de frutos de banana (Musa $A A B$ ) 'Mysore'. $104 \mathrm{f}$. Tese (Doutorado) Universidade Federal de Viçosa, MG.

Sampaio, V.R.; Simão, S. 1970. Banana-ensacamento de cachos logo após o florescimento. Revista de Agricultura, 45(2/3): $75-77$

Santos, J.E.S.; Chitarra, M.I.F. 1998. Relação entre idade do cacho de bananeira "prata", a colheita e a qualidade do fruto após a colheita. Pesquisa Agropecuária Brasileira, 33(9): 1475-1480.

Scarpare Filho, J. A. 1996. Estudo do primeiro ciclo produtivo da bananeira 'Nanicão' (Musa sp. AAA) a partir de diferentes tipos de mudas. Piracicaba, $72 \mathrm{f}$. Tese (Doutorado) - Escola Superior de Agricultura "Luiz de Queiroz", Universidade de São Paulo, Piracicaba, SP.

Soto Ballestero, M. 1992. Bananos: cultivo y comercialización. San José: Litografia e Imprenta LIL, 674p.

Soto Ballestero, M.; Soto, E.; Solis, P.; López, A. 1992. Siembra e operaciones de cultivo. In: Soto Ballestero, M. (Ed.). Banano: cultivo y comercialización. Litografia e Imprenta LIL. San José. p.211-365.

Stover, R.H., Simmonds, N.W. 1987. Bananas. Longman. New York. $468 \mathrm{p}$.

\section{RECEBIDO EM 01/04/2004 ACEITO EM 18/11/2005}

